\title{
MeditAid: A Wearable Adaptive Neurofeedback- based System for Training Mindfulness State
}

\author{
Corina Sas \\ School of Computing and Communications, Lancaster University, UK \\ corina@comp.lancs.ac.uk \\ Rohit Chopra \\ School of Computing and Communications, Lancaster University, UK \\ r.chopra@lancaster.ac.uk
}

\begin{abstract}
A recent interest in interaction design is towards the development of novel technologies emphasizing the value of mindfulness, monitoring, awareness, and self-regulation for both health and wellbeing. Whereas existing systems have focused mostly on relaxation and awareness of feelings, there has been little exploration on tools supporting the self-regulation of attention during mindfulness sitting meditation. This paper describes the design and initial evaluation of MeditAid, a wearable system integrating electroencephalography (EEG) technology with an adaptive aural entrainment for real time training of mindfulness state. The system identifies different meditative states and provides feedback to support users in deepening their meditation. We report on a study with 16 meditators about the perceived strengths and limitations of the MeditAid system. We demonstrate the benefits of binaural feedback in deepening meditative states, particularly for novice meditators.
\end{abstract}

Mindfulness meditation, Mindfulness training, Wearable EEG, Neurofeedback, Aural entrainment

\section{Introduction}

Mindfulness meditation is a practice with significant benefits for health and wellbeing [13] and in particular for the management of stress [35], depression [5,20,81], and chronic pain [34,92]. The skill of maintaining one's attention to the present moment is fundamental to mindfulness meditation [7], yet challenged by the tacit quality of this experience. Meditation is experienced solely from a first person perspective making it difficult to monitor, scrutinize and talk about. As a subjective experience lacking a finely-tuned vocabulary for describing it, mindfulness also offers no external cues on one's performance, so that people are often left wondering how well they meditate. While mindfulness skills can be trained [38], the lack of real time feedback on how the meditation session unfolds is one of the most difficult aspects of the practice, hindering its progress.

Despite its considerable challenges and benefits, there has been a paucity of adaptive computer-based tools for mindfulness meditation training. This is surprising given the recent advent of BrainComputer Interaction (BCI) technologies [31,53,57,79], as well as the robust neuroscience findings on short- and long-term cortical changes related to mindfulness meditation and its subjective experience [87]. Some noticeable exceptions include virtual reality tools employing bio- and neurofeedback training $[31,75,88]$ to support relaxation. Arguably, an easier alternative to meditation is offered by entrainment technologies [56] which generate rhythmic stimuli to produce matching brain 
frequencies [46,62]. Most of the few existing tools have focused on relaxation and bodily awareness rather than regulation of attention to the present moment. They have also employed either neurofeedback or brain entrainment technologies despite the advantage of integrating them [76].

The work presented in this paper introduces MeditAid prototype as an exploration aid for the design space of mindfulness meditation technologies. We explore the potential strengths and limitations of audio entrainment-based neurofeedback for training mindfulness meditation skills, and in particular self-regulation of attention. As a technology for mind-body intervention, MeditAid aligns with the embodiment perspective and uses sound to help people identify different states of meditation]. The system was tested with 16 meditators, in a study designed to explore the role of different types of aural feedback and meditators' expertise on objective and subjective measures of mindfulness' performance. The paper specifically focuses on the following research questions:

1. How effective is the audio entrainment in mindfulness training?

2. How do binaural beats and monaural beats compare in their value for mindfulness training?

3. Do novice meditators benefit more from such training than expert meditators?

4. Can mindfulness state be subjectively measured?

5. Which are the perceived strengths and limitations of MeditAid prototype?

6. Are there any larger design implications for mindfulness training technologies?

The next section offers a review of related work introducing mindfulness meditation, neurofeedback-based mindfulness technologies and the concept brain entrainment. The subsequent section describes the MeditAid system, followed by the presentation of the evaluation study. The outcomes are furthermore discussed in the light of the research questions, and the paper concludes with several implications for design for mindfulness training technologies.

\section{Related Work}

\subsection{Mindfulness Meditation}

Inspired from Eastern meditative practices, mindfulness meditation such as Zen and Vipassana is the process of bringing one's attention to the present moment with a detached awareness of the ever changing perceptual field [77], and an attitude of non-judgmental acceptance of its perceptual content [34]. Self-regulating attention to the present moment means that when aware of the mind wandering into past or future, one takes notice and gently returns attention to the present moment [70]. Assuming the role of an observer of the naturally arising thoughts, and resisting to further elaborate them, ensures a detached awareness from such thoughts [2,33]. This facilitates the stillness of the mind and the awareness that all stimuli are ever changing and transient [68]. The attitude of non-judgmental acceptance applies to both external, social-environmental stimuli, as well as internal stimuli such as bodily sensations, emotions, and thoughts as they arise and are observed without any evaluative judgment [2,51]. Mindfulness meditation could be practiced during ordinary daily activities such as walking or eating [41], or as a sitting practice [36].

Mindfulness meditation has received increasing attention through two wide-spread interventions placing mindfulness training at their core: mindfulness-based stress reduction (MBSR) and mindfulness-based cognitive therapy (MBCT). MBSR was developed by Kabat-Zinn [34] at the 
University of Massachusetts Medical School in late 70s. Designed to support patients with chronic pain and stress-related disorders, MBSR consists of weekly 2 hours group training, and daily 45 minutes individual training over an 8 week course. The program also includes exercises for bodily awareness and discussions about stress and coping mechanisms. MBCT was developed on the base of the MBSR program, with the additional integration of elements of cognitive therapy [81]. The aim here is to facilitate the awareness of the ever changing nature of one's thoughts, feelings and bodily sensations. Just like MBSR, MBCT also consists of an 8 week group program designed to prevent depressive relapse $[5,20,81]$.

Mindfulness meditation and its most common interventions have been shown to lead to significant benefits for stress, wellbeing, physical and mental health in both clinical and non-clinical population $[13,20]$. In a meta-analytic review study, Baer and colleagues [5] showed that these interventions are helpful in the treatment of several disorders. Matching the original aim of each intervention program, Baer's findings confirmed that MBSR leads to significant reduction in ratings of pain and stress, while MBCT significantly reduces the depressive relapse. These benefits of mindfulness interventions for health and wellbeing appear to be mediated by increases in mindfulness [13]. Findings also suggest that a key skill enabling these benefits is the intentional self-regulation of attention from moment to moment $[2,34]$. Increase in the ability to sustain broad attention is fundamental in enabling a more mindful response rather than a habitual one $[2,41]$.

Mindfulness meditation can be conceptualized as both a state and a trait. State refers to changes in sensorial and cognitive awareness arising during the practice, while trait refers to the lasting changes persisting beyond the meditation practice [11]. Assessing the benefits of mindfulness interventions involves measuring the mindfulness construct, either as a trait stable across time and situations, or as a state reflecting changes during the practice situation [14]. In a recent review of mindfulness measurement scales, Chiesa [15] identified several questionnaires measuring the mindfulness trait, which capture either the overall construct of mindfulness, (i.e. the Mindful Attention Awareness Scale (MAAS) [8], the Freiburg Mindfulness Inventory (FMI) [9], the Cognitive and Affective Mindfulness Scale (CAMS) Revised [42]), or its multi-dimensional components such as Kentucky Inventory of Mindfulness Skills (KIMS) [4,5] which includes subscales on non-reactivity, acting with awareness, describing, and non-judging.

In contrast, tools measuring mindfulness state have been less explored. A noticeable exception is the Toronto Mindfulness Scale (TMS) [45] capturing attention to experience with an open nonjudgmental attitude as informed by Bishop's mindfulness theory [7]. The questionnaire was developed and tested with regard to the state mindfulness experienced during the practice of mindfulness meditation. The scale shows good internal consistency and two factors, i.e. curiosity and decentring. Although the scale was validated, the two identified factors failed to capture the main component of mindfulness according to Bishop's theory [7], namely the active selfregulation of attention to immediate experience. Another limitation of the current mindfulness scales is their narrow testing for criterion validity. For example, TMS uses expertise level but no prior work has explored the EEG-based meditative states as validity criterion, despite robust findings on the neurocorrelates of mindfulness state and trait outlined in the next section. 
To summarize, mindfulness meditation is a practice with a wealth of benefits for one's health and wellbeing, which has been intensively explored, with most work focusing on subjective measurements of mindfulness trait. Our paper explores the value of self reports in measuring mindfulness state and aims to validate them with objective measures of mindfulness as reflected in EEG data.

2.1.1 Neurocorrelates of Mindfulness Meditation

As changes in the central system have been consistently found as the most reliable indicator of meditative states, they form the focus of our paper, and in particular changes occurring during meditation practice, i.e. mindfulness state. Such changes in the central system have been traditionally measured in lab settings with EEG technologies involving 24 electrodes placed directly on the scalp. The rhythmic frequency of electrical activity within brain areas are captured by wavebands, measured in number of waves per second, or Hertz, which tend to be associated with specific psychological states $[18,79]$. The five types of rhythms of the brain and their approximate frequency boundaries are delta $(<4 \mathrm{~Hz})$ matching deep sleep, theta $(4-7 \mathrm{~Hz})$ for deeper relaxation and meditation, alpha $(8-14 \mathrm{~Hz})$ for relaxation and meditation, beta $(15-30 \mathrm{~Hz})$ for wakeful state of cognitive activity and anxious thinking, and gamma, $(>30 \mathrm{~Hz})$ for REM sleep, complex cognitive activities, and creativity [18]. Previous work exploring the relationship between EEG frequency and performance on cognitive tasks also indicates that theta activity relates to memory performance, i.e. recognition and working memory; lower alpha to attentional processes, vigilance and creativity, upper alpha to memory retrieval, semantic memory, as well as perceptual and cognitive tasks $[18,39]$.

With respect to neurocorrelates of mindfulness state, studies with Zen masters (20 years of practice) have indicated that they experience the following stages of EEG changes during meditation: (i) appearance of alpha waves ( $\sim$ within the first minute), (ii) increase in amplitude of alpha waves ( $\sim$ within 2-8 minutes), (iii) decrease of frequency of alpha waves ( $\sim$ after 24 minutes from the start), and possibly (iv) appearance of theta waves (after 27 minutes from the start) [36].

Additional studies exploring EEG changes on a moment by moment basis [49] have shown an overall EEG lowering during meditation, from beta to lower alpha and theta activation $[10,11,79,83]$. The lowering of the alpha rhythm has been linked to increase in internal attention, and the increase in the theta band to relaxation, especially within mindfulness meditation [21]. These are significant findings indicating that there are measurable correlates of the subjective experience of mindfulness state.

The impact of mindfulness meditation on long lasting EEG changes, i.e. mindfulness trait, is cumulative as meditators progress in their practice. Fell and colleagues [21] indicated that novices show evidence for slowing alpha rhythms only, while expert meditators show also increase in their theta and gamma rhythms. In addition, a wealth of studies suggested that mindfulness meditation leads to changes in the central and autonomic nervous system [84], triggering long-term changes in the anterior cingulate cortex and dorsolateral prefrontal areas of advanced meditators [11,49].

Our paper focuses on the design, development and evaluation of a prototype aimed to support the training of mindfulness state throughout meditation practice. 


\subsection{Biofeedback- and Neurofeedback-based Mindfulness Training}

Key to mindfulness meditation practice is the development of the ability to self-monitor one's attention and maintain it disengaged from external or internal distractions [2,7,50]. Biofeedback training enables people to learn control their physiological responses. The main outcome of biofeedback training is greater awareness of one's bodily reactions, from heart rate to skin conductance and even brainwaves. The latter is trained through neurofeedback, which is a particular form of biofeedback.

Neurofeedback training involves recording EEG measurements and feeding them back to the individual through audio or visual stimuli, with the aim to support awareness of one's cortical activity and ability to modify it. The learned change of one's neural signal through feedback is key to this training [6]. Changes in cortical activity concurrent with the goal of the training are acknowledged in real time during the training session, and monitored after the session if their impact is expected to last [87]. A recent review by Rabipour and Raz [65] suggests that neurofeedback may be effective for cognitive functions and psychiatric disorders, with earlier studies already indicating its effectiveness for attention deficit disorders [10]. Given the relationship between alpha and theta frequencies on the one hand, and attentional processes and relaxation on the other hand, alpha and theta frequencies appear to be the best candidates for the neurofeedback training in meditation [36].

Despites its benefits, one of the challenges of neurofeedback training is that it requires implicit learning about how to regulate one's brain activity based solely on (visual) feedback on brain's activity $[6,16]$. This occurs in the absence of any aiding tools. One way to address this limitation is to provide aids such as rhythmic stimuli of different modalities.

\subsection{Binaural beats}

Binaural beats are perceived sounds generated by two tones with the same intensity but slightly different frequency delivered separately to each ear. In contrast, monaural beats are generated by two tones with identical frequency delivered at both ears. The two types of beats differ not only in the subjective perception of sound they entail, by also in their underlying biological mechanisms. While monaural beats are due to the stimulation of the inner ear membrane, the binaural beats are made possible by the integration occurring in cortical areas [59,60]. In both cases, the brain perceives a new beat with a frequency half way between the two input tones, and varies in amplitude at a rate equal to the difference between them [16,32]. This modulated signal is perceived when the difference in frequency between the two carrier tones ranges from 2 to $30 \mathrm{~Hz}$ [58,59], and when the individual carrier tones range from 200 to $900 \mathrm{~Hz}$ [64].

Consistent findings have indicated that binaural beats many psychological and physiological processes [2,85] leading to improved relaxation [32,76]., memory, vigilance, concentration [25], attention and mood [44], creativity [66]. Previous findings also suggest that entrainment could be beneficial for attention deficit symptoms, depression, anxiety and pain [76]. In a review of over 20 studies, Huang and colleagues [32] explored the impact of binaural beats on psychological outcomes such as cognition, stress, anxiety, pain relief and mood. Their findings suggest that 
entrainment is an effective therapeutic tool. In a meta-analysis of 16 studies, Pigott and colleagues [61] showed the benefits of visual and sound rhythmic stimuli on anxiety, depression, and paincontrol, and substance abuse.

While the mechanisms of binaural beats are still debated, a common hypothesis is frequency follow response or brain entrainment. Brain entrainment is a process of training the brain to change its dominant EEG frequency towards the frequency of the external rhythmic stimuli, synchronising its neural activity to binaural beats or other rhythmic stimuli $[16,25,32,76]$.

2.3.1 Binaural beats: Neurocorrelates

Findings supporting entrainment hypothesis have shown that rhythmic stimuli trigger changes in thalamus and neocortex $[2,76]$, with binaural beats leading to changes into the temporal regions of the brain [86]. Specific cortical areas involved in the perception of binaural beats include reticular activation system and the inferior colliculus [66]. In a meta-analysis of 16 studies, Pigott and colleagues [61] showed that entrainment strengthens the same brainwave frequency that corresponds to the light and/or sound stimulation. This is made possible through increased brain metabolism and cerebral blood flow, positive biochemical changes in the brain [61].

Studies also indicated variable changes across traditional EEG frequency ranges. For example, most work has shown the impact of binaural beats on changes in delta, theta and gamma band, while a few others have shown changes in alpha or beta ranges (for a review see [87]). Previous findings also suggest that the EEG changes triggered by entrainment could endure even after one exposure to the stimuli, and this can be strengthened by repeated sessions $[61,85]$.

Previous findings also indicated that in order to be effective, medium term exposure to alpha (10 $\mathrm{Hz}$ ) or beta $(20 \mathrm{~Hz})$ binaural beats is required [87] (longer than 5 minutes [37] and shorter than 20 minutes [27]).

Entrainment has also been suggested as supporting meditation [56], with binaural beats leading to increased power in the theta frequency band in experienced meditators [46]. Entrainment could be used by itself, but is particularly useful in combination with neurofeedback as the entrainment frequencies could be matched to the frequency of neurofeedback training [76].

2.4 Biofeedback, neurofeedback and entrainment technologies

In the field of Human-Computer Interaction (HCI), we have drawn from calm design [90], slow design [24], as well as work biofeedback, neurofeedback and entrainment technologies.

Calm design aims to engage both focused and distributed attention enabling richer interaction with technologies and different forms of contemplating them [48]. Slow design emphasizes the value of deliberately investing more time in the interaction with technology so that people better understand and reflect on its use [28]. For example, slow floor [22] and breath-walk aware system [91] illustrate slow designs for supporting mindfulness walking.

Relevant biofeedback technologies have been developed in the areas of personal informatics [47], virtual reality [88] and embodiment [74]. The emerging area of personal informatics [47] has focused on some of the daily life mindfulness practices such as awareness and reflection on one's emotions, thoughts or behaviours [72]. For example, systems such as Affective Diary [78], 
Affective Health [69], AffectAura [52] or AffectCam [73] involve wearable biosensors and were designed to facilitate reflection on feelings. Another example is the three Spheres of Wellbeing for mindfulness, identity and calming, to support vulnerable women with personality disorders and learning disabilities [82]. Relevant here is the mindfulness sphere - a tangible artefact which captures and reflects people's heartbeats through colourful lights and vibration, allowing thus real time self-awareness. This design was intended to engage women in mindfulness and therapeutic practice while sensitively accounting for their safety and personal meaning. To conclude, the above technologies specifically support increased awareness of one's arousal and reflection on it, but they offer less specific support for self-regulation. Virtual reality technologies augmented with physiological sensors have also been used to deliver engaging experiences during the initial stage of meditation practice. Through monitoring breathing patterns [88] as well as heart rate and galvanic skin response [75], such systems support users to create and control through modulating their bodily responses the virtual world's soundscape and landscape. The benefits of such training include relaxation, and relief from stress, anxiety and pain. Other emerging HCI-related areas that may inform mindfulness technologies are embodiment and mind-body interventions [74]. Prior work exploring embodied metaphors has captured relationships between concrete bodily experiences and cognition [43] as well as their value in interaction design. For example, Antle et al. [1] interviewed experts in music and dance to identify mappings between movement and sound parameters, such as the mapping of the amount of physical movement to volume, i.e. more is loud, less is quiet, speed to tempo, proximity to pitch, and flow to rhythm. Their findings showed that the last three mappings were correctly identified, while the volume was problematic as it could be mapped both to speed and amount of movement.

Neurofeedback technologies in the HCI community have recently started to be explored through wearable brain-computer interfaces (BCI). These include the use of brainwaves as innovative input technique for iPhone apps [12], or within game-based scenarios [55]. Applying an embodied interaction lens, O'Hara and colleagues [57] explored the issue of visibility of EEG-based input through players' use of their bodies for staging performance in social games [53]. Beside the interest in games, wearable EEG technologies have been also explored for meditation practice. For example NeuroSky mindset was used to successfully discriminate mindfulness states during meditation [79]. Another example is Sensorium [31], a system integrating neuro- and bio-feedback to support self-awareness and relaxation. The system uses sound and lights to represent changes in both central and autonomous nervous system through a complex set of embodied metaphors. These include several mappings such as delta frequency to string sounds, theta frequency to vibraphone sounds, alpha rhythm to brightness of a green spot, deeper meditative states to blue color, and shallower states to red color. An initial study involved expert and novice meditators instructed to relax and observe the lights and sounds changed by their bodily responses. Findings showed engagement with the system, and increase in bodily awareness and positive emotions.

In contrast with bio- and neuro-feedback technologies in HC, there has been a more limited focus on the development of entrainment technologies for daily meditation practice. An exception is the 
electronic singing bowl developed at Philips [62] which generates monaural beats. Their findings show that such beats increase alpha power and relaxation.

We build on the HCI work reviewed above on three ways. First, we aim to further the exploration of embodied metaphors to support meditation, by effectively mapping the brain activity to sound pitch during meditation training with aural feedback. Our system MeditAid bears similarities with the personal informatics technologies, as it also takes an embodiment perspective to support selfmonitoring and self-awareness for positive changes in health and wellbeing. It is however unique through the focus on awareness and regulation rather than awareness and reflection, attention rather than arousal, and sound-based embodied metaphors rather than the predominant visual ones.

MeditAid also differs from current neurofeedback technologies. For example, it differs from Sensorium through a focus on mindfulness meditation rather than relaxation; self-regulation of attention rather than just self-awareness of bodily changes; as well as detached observation rather than engagement with the external stimuli generated through neurofeedback. In addition, compared to traditional neurofeedback systems restricted to lab settings, MeditAid is a wearable system offering the advantage of being easily used and integrate in daily meditation practice in the home. It innovatively integrates neurofeedback-based audio entrainment to increase awareness of the mindfulness state. The next section introduces the MeditAid system and its components.

\section{MeditAid System}

Within the space of mindfulness technologies, MeditAid prototype has been built to investigate the impact of binaural beats on mindfulness state experienced during meditation. We looked in particular, at the value of audio entrainment-based neurofeedback for training mindfulness meditation. Our target group was people familiar with mindfulness meditation practice and interested to improve their skills, whether beginners or advanced meditators. The MeditAid system aims to (i) capture brain activity during meditation session unobtrusively and reliably; (ii) be wearable, mobile and comfortable, and (iii) provide adaptive audio entrainment. The Figure 1 shows the systems components and how they interact with each other.

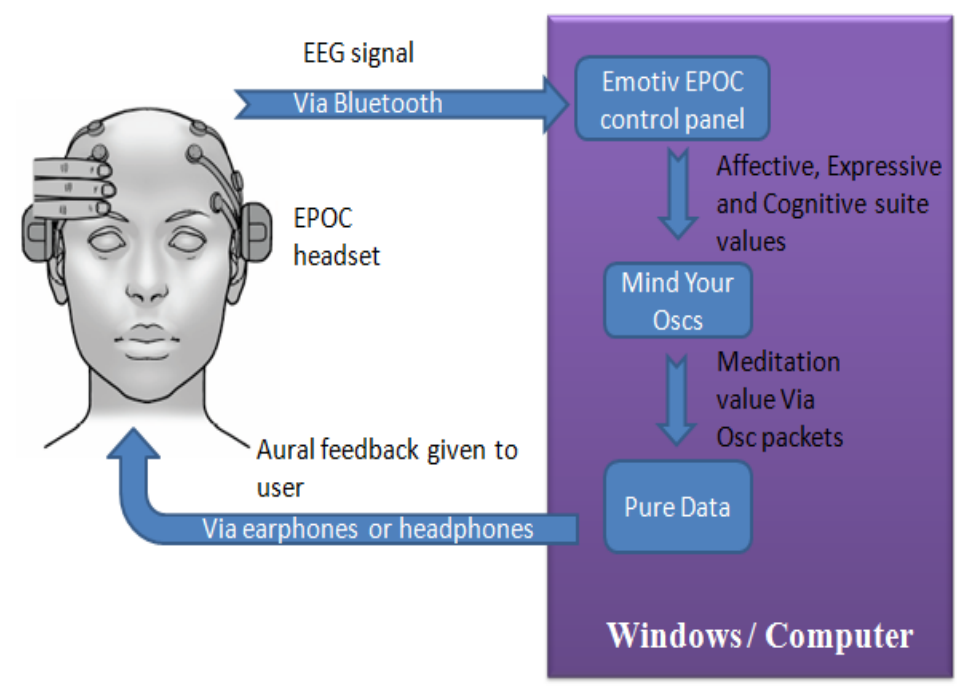

Figure 1: MeditAid System Architecture 
The advent of wearable brain computer interfaces has led to affordable commercial products to emerge on the market. After reviewing the available commercial wireless EEG technology such as Emotiv, Neurosky, OCZ, and Imec, we decided to choose Emotiv. This choice is based on Emotiv offering the larger number of electrodes and arguably more localised accurate readings, i.e. (14 electrodes compared to the 19 electrodes of a standard medical EEG, 10 electrodes of Imec, 7 electrodes of Neurosky, and 3 electrodes of OCZ), as well as an extensive software development kit together with access to developers' forum.

The Emotiv is an affordable wearable device, i.e. \$399. Its portability is ensured by the headset and its fixed sensor-array which is easy to use. The headset approximates the traditional 10-20 positioning scheme for electrodes' placement, AF3/4, F3/4, FC5/6, F7/8, T7/8, P7/8, O1/2 for an average sized head ${ }^{1}$. The frame can be adjusted to fit larger heads while maintaining the placement of the sensors in appropriate locations. The electrodes are placed to one's scalp through saline soaked felt pads. The EEG data is transferred from the headset to a computer through Bluetooth wireless technology.

Previous work has shown that when compared to medical EEG devices, Emotiv records EEG data accurately, making it suitable for non-critical applications [19]. In particular, it can reliably acquire real EEG data and identify meditative states as frequency bands [80]. Emotiv records the electrical signals as a function of time for each electrode of the 14 channels and has a built in digital 5th order Sinc filter ${ }^{2}$. Emotiv's internal sampling rate is $2048 \mathrm{~Hz}$ and after filtering for artefacts with noise cancelation and anti-aliasing methods, it samples at $128 \mathrm{~Hz}$ per channel ${ }^{3}$. Emotiv also filters out frequencies between 50 and $60 \mathrm{~Hz}$ in an attempt to improve signal quality ${ }^{4}$. We used the research edition of the Emotiv Software Development Kit $(\mathrm{SDK})^{5}$ for accessing electricity measurements, as well as the 3D Brain Activity Map application. The latter provides 3D interactive visualization of the brainwave activity across the 4 major bands, i.e. alpha, beta, delta and theta. The 3D Brain Activity Map also provides recordings of EEG real time data filtered through Fast Fourier Transformation ${ }^{6}$. Such data is outputted as edf files which we further processed in MATLAB using the EEGlab tool box. While traditional EEG devices would have provided more accurate readings, the reliability of Emotiv readings couples with its portability and easiness to use made it a preferred contender for the design of MeditAid.

While brain entrainment can be induced through either visual or aural rhythmic stimuli [32], we choose the latter as aural stimuli do not restrict one's attention to the visual display, but broaden it, allowing also for the mindfulness training to take place with closed eyes. Aural feedback has been

\footnotetext{
1 Emotiv. (2014). Electrode Placement. Retrieved 2014, from Emotiv Community: http://www.emotiv.com/forum/forum14/topic25

2 Emotiv. (2014). Extracting RAW data and other applications. Retrieved 2014, from Emotiv Community: http://www.emotiv.com/forum/forum4/topic401/

${ }^{3}$ Emotiv (2014) Sample rate. Retrieved 2014, from Emotiv Community: https://emotiv.com/store/compare/sample rate

${ }^{4}$ Emotiv (2014). Comparison with other EEG units. Retrieved 2014, from Emotiv Community: http://www.emotiv.com/forum/forum4/topic127/

${ }_{5}$ Emotiv. (2014). Developer Edition SDK. Retrieved 2014, from Emotiv Store: $\mathrm{http} / /$ www.emotiv.com/store/sdk/bci/developer-edition-sdk/

${ }^{6}$ Emotiv. (2014). Extracting RAW data and other applications. Retrieved 2014, from Emotiv Community:

$\mathrm{http}: / / \mathrm{www} . \mathrm{emotiv} . \mathrm{com} /$ forum/forum4/topic401/
} 
provided through monaural and binaural beats as they are both beneficial for relaxation, while less work has compared their benefits for mindfulness meditation.

To integrate neurofeedback with aural training, we automatically exported the values of meditative states computed by Emotiv from EEG data, to Pure Data, a free programming language for interactive music and multimedia works. Pure Data extracts the meditation values from the wrapped packets and computes the real time corresponding monaural and binaural beats for each meditation value, subsequently played as aural stimuli to the user's headphones. For example, if the current EEG value is within the alpha band, i.e. $333 \mathrm{~Hz}-432 \mathrm{~Hz}$, MeditAid produces a $12 \mathrm{~Hz}$ binaural beat, i.e. current EEG frequency at right ear, current EEG frequency $+12 \mathrm{~Hz}$ at left ear. For low alpha band, i.e. $433 \mathrm{~Hz}-633 \mathrm{~Hz}$, MeditAid produces a $8 \mathrm{~Hz}$ binaural beat, for theta band, i.e. $634 \mathrm{~Hz}-833 \mathrm{~Hz}$, a $6 \mathrm{~Hz}$ binaural beat, and for delta band, i.e. $>=834 \mathrm{~Hz}$, a $2 \mathrm{~Hz}$ binaural beat. These increments align with previous work on entrainment [23], following closely the frequency of the corresponding band, i.e. delta $(1-3 \mathrm{~Hz})$, theta $(4-7 \mathrm{~Hz})$, alpha $(8-12 \mathrm{~Hz})$. The beats were delivered continuously during the meditation session, with varying frequencies according to meditator's changes in their meditative states.

The delivered beats vary in frequency and subsequently in the perceived pitch. As previously shown, a strong conceptual metaphor related to pitch is vertical height, i.e. up and down [43,63], which may relate to the interaction between pitch and perceived loudness [54]. Thus an appropriate metaphor for mapping the meditative state to the beat was to map the level of meditation to the beat pitch. For this we employed the embodied metaphor of meditation quiets the mind: as meditator progresses through deeper meditative state (or quieter mind), i.e. from beta to alpha, lower alpha, and delta, the binaural bit is perceived as lowering its pitch. Thus the movement towards wakeful state (busy/wandering mind) leads to increased pitch of the beat. In this way, MeditAid system provides both positive feedback for signalling that the user is on the right track, and negative when not. This embodied metaphor aligns with the aliveness quality proposed by Sanches et al. [69], resonating also with previously explored mapping between level of physical activity and sound volume [1].

\section{Study Methodology}

This section focuses on the small scale study exploring the value of MeditAid system in supporting mindfulness state during meditation practice. This initial evaluation tests the usability of the system and people's satisfaction with it. Given the novelty of the technology, such study was considered more appropriate for identifying and addressing any main usability issues before performing a longitudinal study. The following sections describe the study participants, procedure and measurements of performance.

\subsection{Sample}

The sample consisted of 16 participants, 6 males and 10 females (mean age 41, range 20-60). Rather than recruiting from more easily available student population, we chose to recruit from a more difficult to reach population of mindfulness meditation practitioners. Participants were recruited through a convenience sample as active members of two Buddhist meditation groups in the UK. Participants' training in mindfulness meditation ranged from 1 month to 40 years, and half of them had more than 8 years of training. The definition of expertise in meditation practice is not 
commonly agreed upon. However, some previous work on the impact of mindfulness meditation on meditators have also employed 8 years of practice as a cut-off point between novices and experts $[33,67]$.

\subsection{Procedure}

Given the complexity of meditation skills and the difficulty of identifying matching groups, we employed a mixed factorial design with the type of training as within-subject variable, and expertise as a between-subjects variable. The evaluation study has also focused on the use of the system in people's preferred meditation settings rather than in the lab. Participants were introduced to the MeditAid system, the headset was attached to their scalps, and the earphones on. The meditator was then asked to perform three mediation sessions: a control condition with no feedback, an entrainment condition with monaural beats, and an entrainment condition with binaural beats. Each session lasted 10 minutes and the entrainment conditions were counterbalanced. The aural entrainment was delivered a fixed standard volume through stereo headphones to prevent the interference of background noise.

There are few suggestions regarding the most appropriate entrainment's duration, and agreeing with previous suggestions that is should be between 5 and 20 minutes [27,37], we decided to apply 10 minutes per condition. Meditation sessions were interspersed with breaks lasting in average 15 minutes, for gathering participants' feedback about the previous meditation session through semistructured interviews. The interviews captured the following themes: participants' subjective measures of mindfulness experienced during the previous meditation session, perceived usefulness of the system, strengths and limitations, and participants' opinions about how the system could be improved. The interviews were recorded, fully transcribed and analysed for extracting the main themes.

\subsection{Measurements}

Objective measures of mindfulness were gathered during the meditation session through monitoring the EEG patterns using the Emotiv system and identifying the associated meditative states. Subjective measures of mindfulness were captured after each meditation session through interviews. For this we reviewed existing mindfulness scales and considered its key aspects as outlined in the background section. Toronto Mindfulness Scale [45] is one of the few scales measuring state mindfulness. Although developed on the basis of Bishop's theory [7], TMS fails to capture self-regulation of attention to immediate experience. It is precisely the state mindfulness and self-regulation of attention that are relevant to our research questions. Hence, given the gap of a valid and reliable scales capturing it, we designed three items for operationalizing this key mindfulness skill. We asked participants about how well did they succeed in being still during the meditation session, providing a five-point Likert scale for quantifying the answer from 1 (not at all), 2 (to a small extent), 3 (average), 4 (to a large extent) to 5 (completely). Stillness is often used in meditators' vocabulary to describe stable focus of attention. We also asked them how many times they have to come back to the still point, after having engaged in deliberate thoughts. Finally, we asked them to assess the percentage of time during the 10 minutes meditation session when their mind was still. To test the criterion validity of these items we explored their correlation with our objective measures of mindfulness performance. To measure perceived usefulness of the 
system we asked participants to what extent the aural feedback helped them meditate. For these we also provided five-point Likert scales from 1 (not at all), 2 (to a small extent), 3 (average), 4 (to a large extent) to 5 (completely).

\section{Results}

We now revisit the research questions described in the Introduction. In order to explore the effectiveness of the audio entrainment in mindfulness training, we need to look at the measurements of mindfulness state. The means and standard deviations for both objective and subjective measures are presented in Table 1. The objective measure consists of the deepest meditation states reached during each session derived from the EEG frequency bands. For readability we converted the frequency bands into ordinal values, (i.e., beta $=0$, alpha $=1$, lower alpha $=2$, and theta $=3$ ). The subjective measurements were captured by participants' answers on a 5 point Likert scale to the three items we designed.

Table 1. Descriptive data for study participants $(n=16)$

\begin{tabular}{|c|c|c|c|c|c|c|c|c|c|}
\hline & & \multicolumn{2}{|c|}{$\begin{array}{l}\text { Deepest } \\
\text { meditation state } \\
\text { based on } \\
\text { EEG frequency bands }\end{array}$} & \multicolumn{2}{|c|}{$\begin{array}{lr}\text { Mean } & \text { self-rated } \\
\text { success } & \text { in } \\
\text { meditation session }\end{array}$} & \multicolumn{2}{|c|}{$\begin{array}{lr}\text { Mean \% of time of } \\
\text { self- } & \text { reported } \\
\text { stillness } & \end{array}$} & \multicolumn{2}{|c|}{$\begin{array}{l}\mathrm{Nr} \text { of times of } \\
\text { bringing attention to } \\
\text { the present moment }\end{array}$} \\
\hline & Expertise & Mean & $\begin{array}{l}\text { Std. } \\
\text { Dev. }\end{array}$ & Mean & $\begin{array}{l}\text { Std. } \\
\text { Dev. }\end{array}$ & Mean & $\begin{array}{l}\text { Std. } \\
\text { Dev. }\end{array}$ & Mean & $\begin{array}{l}\text { Std. } \\
\text { Dev. }\end{array}$ \\
\hline \multirow[t]{2}{*}{ Control Group } & Novice (8) & 0.83 & 0.75 & 2.67 & 0.52 & 39.17 & 12.01 & 8.20 & 1.64 \\
\hline & Expert (8) & 1.67 & 0.52 & 3.50 & 0.84 & 57.33 & 24.69 & 3.70 & 0.84 \\
\hline Monaural & Novice (8) & 0.83 & 0.75 & 3.00 & 0.63 & 43.33 & 10.80 & 6.70 & 2.17 \\
\hline Entrainment & Expert (8) & 1.83 & 0.41 & 3.50 & 0.84 & 53.50 & 26.30 & 3.50 & 0.71 \\
\hline Binaural & Novice (8) & 1.83 & 0.75 & 3.17 & 0.41 & 46.00 & 15.70 & 6.40 & 1.14 \\
\hline Entrainment & Expert (8) & 2.33 & 0.82 & 3.67 & 0.98 & 65.00 & 28.11 & 3.50 & 0.71 \\
\hline
\end{tabular}

5.1 Objective and subjective measurements of mindfulness state

To explore the effectiveness of audio entrainment, we run a $3 \times 2$ factorial ANOVAs using entrainment and expertise as independent variables with three levels (control condition, monaural entrainment and binaural entrainment), and two levels respectively (novices and experts). The dependent variable was objective measure of performance.

ANOVA findings show both the main effects of the entrainment and expertise, as well as an interaction effect. On average, all meditators, irrespectively of expertise, achieved a significantly deeper level of meditation with binaural feedback, (i.e. as measured by lower alpha) as opposed to monaural feedback or no feedback at all (i.e., as measured by alpha) $\left(F(2,28)=57.84, p<.05, \eta^{2}=\right.$ 0.80). The main effect of expertise indicate that expert meditators, irrespectively of the type of entrainment, achieved a significantly deeper level of meditation (i.e., lower alpha) than novice meditators (i.e., alpha), $\left(F(1,14)=11.19, p<.05, \eta^{2}=0.44\right)$. Finally, the interaction effect suggests that binaural entrainment had a significantly stronger impact on novices as they successfully deepened their meditate state from alpha to lower alpha $\left(F(2,28)=4.79, p<.05, \eta^{2}=\right.$ 0.26). In contrast, experts experienced a smaller benefit within the lower alpha band, although half of them have also reached theta. 
Another research question focused on how can mindfulness state and in particular self-regulation of attention to present moment be subjectively measured? We designed three items potentially tapping into the construct of mindfulness state. If subjective measures of mindfulness are measuring the same construct as the objective measures of mindfulness, then they should correlate. To explore the relationship between the objective and subjective measures of mindfulness, we computed their correlations within each entrainment condition (Table 2). If significant, such correlations could offer valuable criterion validity for our subjective mindfulness measures. This will be an important outcome, given the limited research into the development of scales for measuring state mindfulness.

Table 2: Correlations of objective and subjective measure of mindfulness in each condition

\begin{tabular}{|c|c|c|c|c|}
\hline $\begin{array}{l}\text { Entrainment } \\
\text { Condition }\end{array}$ & $\begin{array}{l}\text { Self-rated } \\
\text { experiencing } \\
\text { mindfulness } \\
\text { session }\end{array}$ & $\begin{array}{c}\text { success of } \\
\text { stillness in } \\
\text { meditation }\end{array}$ & 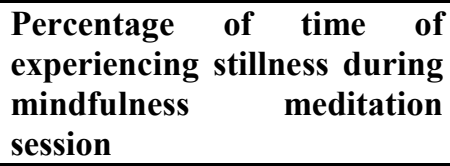 & $\begin{array}{l}\text { Number of times of } \\
\text { bringing attention to the } \\
\text { present moment during } \\
\text { meditation }\end{array}$ \\
\hline $\begin{array}{l}\text { Control } \\
\text { Monaural } \\
\text { Binaural }\end{array}$ & $\begin{array}{l}r(14)=0.55^{*} \\
r(14)=0.40 \\
r(14)=0.47\end{array}$ & & $\begin{array}{l}r(14)=0.54 *\left(\mathrm{R}^{2}=30 \%\right) \\
r(14)=0.51 *\left(\mathrm{R}^{2}=26 \%\right) \\
r(14)=0.55 *\left(\mathrm{R}^{2}=30 \%\right)\end{array}$ & $\begin{array}{l}r(14)=-0.58^{*} \\
r(14)=-0.40 \\
r(14)=-0.38\end{array}$ \\
\hline
\end{tabular}

* Correlation is significant at the 0.05 level (2-tailed).

Findings show that only one subjective measure of mindfulness correlates significantly with the objective measure across all three conditions, i.e. the percentage of time experiencing stillness. For this reason, this is the only subjective measure of performance that we will consider for further analysis. We run a second $2 \times 3$ ANOVA, this time with subjective measure of performance as dependent variable. Findings show a main effect of the type of entrainment on the self-reported percentage of time of experiencing stillness during meditation. Meditators reported successful regulation of attention or present-centered awareness significantly longer when they received binaural feedback ( $55 \%$ of the time) as opposed to monaural feedback or no feedback at all $(47 \%$ of the time), $\left(F(2,28)=8.74, p<.05, \eta^{2}=0.38\right)$. Findings show no main effect of expertise, and no interaction effect.

5.2 Subjective assessment of MeditAid system

We now describe participants' subjective satisfaction with MeditAid measured through the question: to what extent the aural feedback helped them meditate. This was measured on a fivepoint Likert scale for quantifying the answer from 1 (not at all), 2 (to a small extent), 3 (average), 4 (to a large extent) to 5 (completely). As shown in Table 3, participants considered the monaural and binaural feedback above averagely helpful.

Table 3: Descriptive statistics of perceived helpfulness of monaural and binaural beats

\begin{tabular}{lllc}
\hline Condition & Expertise & Mean & Std Dev \\
\hline Monaural beats & Novice & 3.00 & 0.6 \\
& Expert & 3.50 & 0.8 \\
Binaural beats & Novice & 3.20 & 0.4 \\
& Expert & 3.70 & 1.0 \\
\hline
\end{tabular}

The perceived strengths and opportunities for improving the MeditAid as captured through the interviews are further summarized. All meditators identified the benefit of MeditAid in supporting 
attention regulation. With respect to monaural entrainment, experts mentioned that "it was useful because it helped me to recognize my engagement in unwanted thoughts and I easily took my mind off from it and came back to the still point." The value of MeditAid in supporting self-regulation of one's thoughts and monitoring of how meditation progresses, was acknowledged by both novices: "Aural feedback helped me to keep a track of thoughts", and experts: "It enabled me to understand exactly what is going on during the meditation, as well as to keep track of my thoughts".

Novices also mentioned how the higher pitch, associated with higher brain frequencies was used to identify the movement out from deep meditative states to more shallow ones: "As soon as the tone went up I realized that I engaged myself in some thought so it was easy for me to disengage. All I had to do to bring the tone down is to take deep breath and let the thoughts go." The perceived benefits led experts to comment on the value of the MeditAid for mindfulness training: "I think it's a brilliant idea to watch your thoughts. I am a counsellor and people come with problems like: I cannot meditate because I cannot keep a track of my thoughts. I think with such proper training they can help themselves to meditate in an efficient way ...I'm sure it's something should be considered in the market".

The binaural entrainment has led to a sharp distinction in participants' perceived benefits, according to their level of expertise. Thus, experts emphasised that binaural beats allowed them to reach deeper levels of meditation: "it helped me to monitor my meditation and go into the deeper states quickly". Some experts also experienced deep relaxation: "I feel more relaxed after this kind of meditation" or even sleepiness:"I fell asleep and I was informed about my meditation state, so that was interesting... and I feel as if I have just woken up and I feel fresh". In contrast, novices failed to notice significant differences between the monaural and binaural beats: "I didn't experience much difference since last time", although three of them pointed to the sleepiness effect: "I can't feel much difference since last time apart from the fact that I'm a bit sleepy now."

In terms of limitations, both experts and novices noted the distracting nature of the beats and the discomfort of wearing the Emotiv headset and earphones. Some experts mentioned that they may prefer quiet time for meditation "I like to meditate without sound, so it was a bit difficult for me". Novices added that "it is uncomfortable to wear the headset and earphones and meditate" and that "it made me a bit anxious and it was very distracting". Another novice pointed to the raise in self-judgment as a result of mindfulness feedback: "when I see sound going up and down, I am more anxious about keeping it low and then I end up with a fearful thought that I am not meditating properly and I cannot take my mind off from it." Three participants experience mild headache and one expressed concern about the unknown side-effects associated with long term use.

We also explored participants' opinions regarding ways to improve the experience of the system. Experts' suggestions include converting the pitch of the beat either through instrumental or naturebased music: "reduce the amount of high pitch noise and introduce some background music like nice violin, chirping of birds or sea waves". This is particularly useful for people who prefer to meditate with music. Other people suggested that: "it would be good to listen to different tones for different meditation music like guitar for alpha state and waterfall for theta state". In contrast, others prefer quiet meditation without any sound aid: "another way of giving the feedback of 
meditation states without disturbing would be a good job...I find meditating quietly very peaceful and healing." Novices also pointed out to the value of relaxing music: "soothing music will be helpful as sometimes it is difficult to meditate with a tone", and of non-sound meditation aids: "I would love to monitor my meditation and improve it but not through aural feedback. It would be nice to get feedback through other senses". Novices also mention the value of integrating meditation with mobility: "It would be good to have an Iphone application for this device, so that we can link the headset with our phone and make portable."

\section{Discussion}

In this section we discuss the main findings and their implications for the future technologies for training mindfulness state. One of the most important finding is that meditators successfully achieved significantly deeper meditation states with adaptive aural entrainment and in particular binaural beats, i.e. lower alpha, compared to monaural beats and control condition, i.e. alpha. This provides support for the entrainment hypothesis, particularly in alpha band. Such outcome is particularly important given the limited duration of the mindfulness meditation training, i.e. 10 minutes. Previous work identified that experienced Zen masters with over 20 years of mindfulness meditation practice need over 8 minutes of meditation to start lowering alpha frequency [36]. In addition, the two most common interventions for mindfulness training, MBSR and MBCT involve about 45 minutes daily training over an 8 weeks course [20,34,81]. Thus, our findings suggest that mindfulness technologies could potentially reduce the length of training to self-regulate one's attentional skills, particularly for novice meditators. This in turn could allow for quicker access to the rich benefits of mindfulness for health and wellbeing [29, 93].

Another finding shows the importance of expertise in mindfulness meditation when exploring the impact of the MeditAid prototype. Given that relationship between the length of practice and the long term changes in meditators' central nervous system [84], it was expected that experts achieved significantly deeper meditative states compared to novices. Findings confirm that experts' outperform novices irrespectively of the entrainment condition. What is important is that compared to experts, novice meditators experienced a significantly larger increase in their mindfulness' performance successfully deepening their meditate state from alpha to lower alpha. This finding suggests that even a brief training session of mindfulness meditation with binaural entrainment is highly beneficial for novice - those in more need to learn to regulate their attention. In turn, this could particularly support novices reduce their learning curve to more successfully reap the benefit of mindfulness [29,93].

Our findings also indicate that MeditAid's adaptive binaural beats support self-regulation of attention in mindfulness meditation state, when compared to monaural beats. The increased benefits of binaural entrainment in mindfulness training can be placed within those findings indicating the benefits of binaural beats on relaxation, health and wellbeing (for a review see [2]). This may be due to the different physiological mechanisms of monaural and binaural beats, i.e. stimulation of the inner ear membrane vs cortical integration [59,60]. An interesting observation is the interaction between pitch and perceived loudness, with previous studies showing that higher pitched sounds are perceived as louder [54]. The less effective monaural entrainment meant that 
people were exposed more to higher pitched beats than during binaural entrainment. This in turned has colored the perception of monaural feedback as louder and more distractive than binaural one. One way of addressing this is using binaural beats or allowing the users to set lower volume settings for their feedback.

The identified benefits of audio entrainment include support for self-regulating one's attention, recognizing one's engagement in deliberate thoughts, and shifting one's attention to the present moment. Such benefits were experienced by both experts and novices, in both monaural and binaural condition. In addition, experts identified binaural beats as even more conducive to deeper meditation states, relaxation or sleepiness.

Another contribution of our work is the exploration of the three novel subjective measures of mindfulness state. Findings show that only the percentage of time of experiencing stillness appears to be valid measure. The small size effect of its correlation with objective measures of mindfulness state suggests that future work is still required in this area.

\section{Design Implications}

We now turn our attention to the larger implications of these findings for this new class of technologies for training mindfulness in real life. Our findings suggest that mindfulness training could well benefit from integrating BCI and entrainment technologies. Previous attempts of exploring these two classes of technologies for mindfulness training have been limited both in number and scope. The MeditAid prototype and its effectiveness for mindfulness meditation open new opportunities for the use of $\mathrm{BCI}$ beyond game design, and towards increased self-regulation.

7.1 Support for self-regulation of attention

Within the field of HCI, our prototype positions itself at the intersection of several emerging areas: the field of personal informatics with technologies for self-monitoring and self-awareness of emotions. The latter builds heavily on the biofeedback principle, although systems providing realtime support are rare $[52,69,73,78]$. It also contributes to the increasing interest in embodiment and somatics [74] and technologies for mind-body interventions. Our system bears similarities with such technologies, as it supports self-monitoring, self-awareness and positive change for health and wellbeing. However unlike existing work focusing on regulating autonomic arousal, i.e. Affective Health [69], AffectAura [52], MeditAid innovatively explores the self-regulation of attention. This focus is an important design principle for mindfulness technologies because the ability to actively self-regulate attention to immediate experience and disengage from distracting stimuli is a key skill in mindfulness practice [2, 7, 50]. Moreover, it bears links with the field of personal informatics and technologies for emotional awareness and affect regulation, since attention regulation is an important prerequisite for developing awareness, distance and nonjudgmental acceptance of present emotions [29]. Thus when designing for emotional awareness and regulation, designers could benefit from considering also the value of attention regulation addressed within the larger space of mindfulness technologies.

6.2 Low volume, discrete binaural feedback

As a feedback technology, MeditAid has the potential to help by increasing people's awareness of their movement in and out of deeper meditative states. Allowing users control over the volume of 
binaural beats is particularly important. An important design implication concerns novice meditators as they are at higher risk of sleeping out of deeper meditative states and become judgmental with respect to their success in mindfulness practice. Providing discrete rather than continuous binaural feedback, for example only after the meditator has been in beta range for a set time interval, i.e. 5 minutes, can help limit this risk and offset novices' self judgment.

6.3 Haptic entrainment

While current entrainment technologies involve predominantly rhythmic visual or aural stimuli $[16,32,76]$, our findings suggest that aural feedback can add to distraction, as people strongly prefer quietness during meditation. While haptic actuators have started being used to support bodily awareness [26], the value of rhythmic haptic stimuli has been less explored in entrainment $[32,89]$. For example haptic actuators could be embedded in thimbles to be used on meditators' thumbs. This is supported by the semiotics of hand, fingers' positions and their role in mediation. One such position involves the dominant hand holding the other hand with the thumbs touching lightly forming an oval. Historically, the role of the touching of the thumbs is precisely the selfmonitoring of attention; when they are pressed there is an indication of stress or mind wandering, and when the touch is lost there is an indication of loss of concentration or falling asleep. Thus future mindfulness technologies could open up this design space of haptic entrainment harnessing rich embodied metaphors mapping meditative states to touch such as "pressing too hard and getting stressed".

\subsection{Embodied metaphorical learning}

Since neurofeedback training does not provide explicit instructions as to how the brain activity may be controlled, this ability appears to be acquired not explicitly but rather through implicit learning $[6,16]$. The link between embodiment and implicit self-regulation in meditation practice has also been suggested, for example through posture and breathing patterns [40]. In addition, findings in embodied cognition have long acknowledged the value of embodied metaphors capturing relationships between concrete bodily experiences and cognition [43]. Our metaphor "meditation quiets the mind" worked well as repeatedly expressed in participants' answers where no mapping errors were reported.

\section{Conclusions}

Our study explored the benefits and limitations of MeditAid as a meditation aid for mindfulness state. Study findings showed the value of aural feedback in deepening the meditative states, particularly for novice meditators. We advance mindfulness theory with subjective measure of state mindfulness whose validity is supported by the correlation with objective mindfulness measures. Our findings also led to a number of design implications for mindfulness meditation including support for self-regulation of attention and haptic feedback, as well as the value of several qualities of embodied metaphors. The MeditAid prototype and its effectiveness for mindfulness meditation open new opportunities for the use of Brain Computer Interaction technologies beyond game design, and towards increased self-regulation. Such tools could also be integrated with the most common intervention programs for mindfulness training, i.e. MBSR and MBCT. Future work will explore how the MeditAid's benefits could extend to these programs, and longitudinal studies could investigate MeditAid's value in supporting mindfulness trait. We hope 
that this work will inspire novel systems and applications exploiting the untapped potential of BCI for health and wellbeing.

\section{Acknowledgements}

The authors would like to thank study participants.

\section{References}

1. Antle, A. N., Corness, G., Droumeva, M. (2009). What the body knows: Exploring the benefits of embodied metaphors in hybrid physical digital environments. Interacting with Computers, 21(1), 66-75.

2. Atwater, F.H. 2001. Binaural beats and the regulation of arousal levels. In Proc. of the TANS 11 Forum on New Arts and Science. (18 pages).

3. Baer RA (2003) Mindfulness training as a clinical intervention: a conceptual and empirical review. Clin Psychol Sci Pract 10(2):125-143

4. Baer, R.A., Smith, G.T., Allen, K.B. 2004. Assessment of mindfulness by self-report: The Kentucky inventory of mindfulness skills. Assessment, 11, 191-206.

5. Baer, R.A., Smith, G.T., Hopkins, J., Krietemeyer, J., Toney, L. 2006. Using self-report assessment methods to explore facets of mindfulness. Assessment, 13, 27-45.

6. Birbaumer, N., Ruiz, S., Sitaram, R. 2013. Learned regulation of brain metabolism. Trends in cognitive sciences.

7. Bishop, S.R., Lau, M., Shapiro, S., Carlson, L., Anderson, N. D., Carmody, J. 2004. Mindfulness: A proposed operational definition. Clinical Psychology, 11, 230-241.

8. Brown, K.W., Ryan, R.M. 2003. The benefits of being present: Mindfulness and its role in psychological wellbeing. Journal of personality and social psychology, 84, 822-848.

9. Buchheld, N., Grossman, P., Walach, H. 2001. Measuring mindfulness in insight meditation (Vipassana) and meditation based psychotherapy: The development of the Freiburg Mindfulness Inventory (FMI). Journal for meditation and meditative research, 1, 11-34.

10. Butnik, S.M. 2005. Neurofeedback in adolescents and adults with attention deficit hyperactivity disorder. Journal of Clinical Psychology, 61(5), 621-625.

11. Cahn B.R. and Polich, J. 2006. Meditation states and traits: EEG, ERP, and neuroimaging studies. Psychological Bulletin 132 (2): 180-211.

12. Campbell, A., Choudhury, T., Hu, S., Lu, H., Mukerjee, M.K., Rabbi, M. and Raizada, R.D.S. 2010. NeuroPhone: brain-mobile phone interface using a wireless EEG headset. In Proc. of the second ACM SIGCOMM workshop on Networking, systems, and applications on mobile handhelds (MobiHeld '10). ACM, New York, NY, USA, 3-8.

13. Carmody, J., Baer, R.A. 2008. Relationships between mindfulness practice and levels of mindfulness, medical and psychological symptoms and well-being in a mindfulness-based stress reduction program. Journal of behavioral medicine, 31(1), 23-33.

14. Chiesa A, Serretti, A. 2010. A systematic review of neurobiological and clinical features of mindfulness meditations. Psychological Medicine 40 (8): 1239-1252.

15. Chiesa, A. 2013. The difficulty of defining mindfulness: current thought and critical issues. Mindfulness, 4(3), 255-268.

16. Cho, M.K., Jang, H.S., Jeong, S.H., Jang, I.S., Choi, B.J., Lee, M. (2008). Alpha neurofeedback improves the maintaining ability of alpha activity. Neuroreport, 19(3), 315-317.

17. Collura, T. Siever, D. 2009. Audio-visual entrainment in relation to mental health and EEG. In J.R. Evans \& A. Abarbanel (Eds.) Quantitative EEG and Neurofeedback (2nd Ed.) (pp. 155-183) San Diego, CA: Academic Press.

18. Dooley, C. 2009. The impact of meditative practices on physiology and neurology: a review of the literature. Scientia Discipulorium, 4, 35-59.

19. Duvinage, M., Castermans, T., Petieau, M., Hoellinger, T., Cheron, G., \& Dutoit, T. (2013). Performance of the Emotiv Epoc headset for P300-based applications. Biomedical engineering online, 12(1), 56.

20. Evans, S., Ferrando, S., Findler, M., Stowell, C., Smart, C., Haglin, D. (2008). Mindfulnessbased cognitive therapy for generalized anxiety disorder. Journal of anxiety disorders, 22(4), 716.

21. Fell, J., Axmacher, N., Haupt, S. 2010. From alpha to gamma: Electrophysiological correlates of meditation-related states of consciousness. Medical hypotheses, 75(2), 218-224.

22. Feltham, F., Loke, L. 2012. The Slow Floor: towards an awareness of bodily movement through interactive walking surfaces. In Proc. of OZCHI 2012, 12-16. 
23. Foster, D. S. (1990). EEG and subjective correlates of alpha frequency binaural beats stimulation combined with alpha biofeedback (Doctoral dissertation, Memphis State Univ.).

24. Fuad-Luke, A. 2005. Slow Theory; A paradigm for living sustainably?

25. Gao, X., Cao, H., Ming, D., Qi, H., Wang, X., Wang, X., ... \& Zhou, P. (2014). Analysis of EEG activity in response to binaural beats with different frequencies. International Journal of Psychophysiology, 94(3), 399-406.

26. Giannoulis, S., \& Sas, C. (2013). VibeRate, An Affective Wearable Tool for Creative Design. In Proc. IEEE Affective Computing and Intelligent Interaction (ACII), 2013 Humaine Association Conference, 594-599. IEEE.

27. Goodin, P., Ciorciari, J., Baker, K., Carrey, A., Harper, M., \& Kaufman, J. (2012). A high density EEG investigation into steady state binaural beat stimulation. PLoS One, 7(4),

28. Grosse-Hering, B., Mason, J., Aliakseyeu, D., Bakker, C., and Desmet, P. 2013. Slow design for meaningful interactions. In Proc. of the Conference on Human Factors in Computing Systems, 3431-3440. ACM.

29. Grossman, P., Niemann, L., Schmidt, S., Walach, H. 2004. Mindfulness-based stress reduction and health benefits. A meta-analysis. Journal of psychosomatic research, 57(1), 35-44

30. Hayes, A.M., Feldman, G. 2004. Clarifying the construct of mindfulness in the context of emotion regulation and the process of change in therapy. Clinical Psychology: Science and Practice, 11(3), 255-262.

31. Hinterberger, T. 2011. The sensorium: a multimodal neurofeedback environment. Adv. in Hum.-Comp. Int. Article 3, 10 pages.

32. Huang, T.L., Charyton, C. 2008. A comprehensive review of the psychological effects of brainwave entrainment. Altern Ther Health Med, 14(5), 38-50.

33. Ivanovski, B., \& Malhi, G. S. (2007). The psychological and neurophysiological concomitants of mindfulness forms of meditation. Acta neuropsychiatrica, 19(2), 76-91.

34. Kabat-Zinn, J. 1982. An outpatient program in behavioral medicine for chronic pain based on the practice of mindfulness meditation. General Hospital Psychiatry, 4, 33-47.

35. Kabat-Zinn, J. 1994. Wherever you go, there you are. New York: Hyperion.

36. Kasamatsu, A., Hirai, T. 1966. An electroencephalographic study on the Zen meditation (Zazen). Psychiatry and Clinical Neurosciences, 20(4), 315-336.

37. Kennerly, R. (1996). An empirical investigation into the effect of beta frequency binaural beat audio signals on four measures of human memory. Hemi-Synch Journal, 14(3), 1-4.

38. Kerrigan, D., Johnson, K., Stewart, M., Magyari, T., Hutton, N., Ellen, J. M., Sibinga, E. 2011. Perceptions, experiences, and shifts in perspective occurring among urban youth participating in a mindfulness-based stress reduction program. Complementary Therapies in clinical practice, 17(2), 96-101.

39. Klimesch, W. 1999. EEG alpha and theta oscillations reflect cognitive and memory performance: A review and analysis. Brain Research Reviews, 29 (2-3), 169-195.

40. Koole, S.L., McCullough, M.E., Kuhl, J., Roelofsma, P.H. 2010. Why religion's burdens are light: From religiosity to implicit self-regulation. Personality and Social Psychology Review, 14(1), 95-107.

41. Kristeller, J.L. 2007. Mindfulness meditation. In P. Lehrer, R. Wookfolk \& W. E. Simes (Eds.), Principles and practices of stress management, pp. 393- 427. New York, NY: Guilford Press.

42. Kumar, S.M., Feldman, G.C., Hayes, S.C. 2008. Changes in mindfulness end emotion regulation in an exposure based cognitive therapy for depression. Cognitive Therapy \& Research, 32, 734-744.

43. Lakoff, G., Johnson, M., 1980. Metaphors We Live By. Chicago Press, Chicago, IL, US.

44. Lane, J. D., Kasian, S. J., Owens, J. E., \& Marsh, G. R. (1998). Binaural auditory beats affect vigilance performance and mood. Physiology \& behavior, 63(2), 249-252.

45. Lau, M.A., Bishop, S.R., Segal, Z.V., Buis, T., Anderson, N.D., Carlson, L., Devins, G. 2006. The Toronto mindfulness scale: Development and validation. Journal of clinical psychology, 62(12), 1445-1467.

46. Lavallee, C.F.; Koren, S.A., Persinger, M.A. 2011. A Quantitative Electroencephalographic Study of Meditation and Binaural Beat Entrainment. Journal of Alternative and Complementary Medicine, 17 (4): 351-355.

47. Li, I., Dey, A. K., Forlizzi, J. (2011). Understanding my data, myself: supporting self-reflection with ubicomp technologies. In Proc. of Ubiquitous computing, 405-414. ACM.

48. Linn, S., Kelliher, A. 2010. Speak More Slowly! Developing better community awareness through localized reflective media systems. In Proc. of IDSA 2010 National Conference. 
49. Lutz, A., Greischar, L. L., Rawlings, N. B., Ricard, M., \& Davidson, R. J. 2004. Long-term meditators self-induce high-amplitude gamma synchrony during mental practice. In Proc. of the National Academy of Sciences of the United States of America, 101(46), 16369-16373

50. Lutz, A., Slagter, H. A., Dunne, J. D., \& Davidson, R. J. 2008. Attention regulation and monitoring in meditation. Trends in cognitive sciences, 12(4), 163.

51. Marlatt, G.A., Kristeller, J.L. 1999. Mindfulness and meditation. In W. R. Miller (Ed.), Integrating spirituality into treatment (pp. 67-84). Washington, DC: APA.

52. McDuff, D., Karlson, A., Kapoor, A., Roseway, A., Czerwinski, M. 2012. AffectAura: an intelligent system for emotional memory. In Proc. of the 2012 ACM annual conference on Human Factors in Computing Systems, 849-858). ACM.

53. Nacke, L.E., Kalyn, M., Lough, C. and Mandryk, R.L. 2011. Biofeedback game design: using direct and indirect physiological control to enhance game interaction. In Proc. of the SIGCHI Conference on Human Factors in Computing Systems (CHI '11). ACM, 103-112.

54. Neuhoff, J. G., Wayand, J., \& Kramer, G. (2002). Pitch and loudness interact in auditory displays: Can the data get lost in the map? Journal of Experimental Psychology: Applied, 8(1), 17. 55. Nijholt, A., Tan, D., Allison, B., Milan, J. and Graimann, B. 2008. Brain-computer interfaces for HCI and games. In Proceedings of CHI '08 Extended Abstracts on Human Factors in Computing Systems (CHI EA '08). ACM, New York, NY, USA, 3925-3928.

56. Norhazman, H., Zaini, N.M., Taib, M.N., Omar, H.A., Jailani, R., Lias, S., Sani, M.M. (2012), Behaviour of EEG Alpha Asymmetry when stress is induced and binaural beat is applied. In Computer Applications and Industrial Electronics, IEEE Symposium, 297-301. IEEE.

57. O'Hara, K., Sellen, A. and Harper, R. 2011. Embodiment in brain-computer interaction. In Proceedings of the SIGCHI Conference on Human Factors in Computing Systems (CHI '11). ACM, New York, NY, USA, 353-362.

58. Oster, G. 1973. Auditory beats in the brain. Scientific American, 229(4), 94-102.

59. Perrott, D. R., \& Nelson, M. A. (1969). Limits for the detection of binaural beats. Journal of the Accoustical Society of America, 46, 1477-1481.

60. Peterson, J. (1916) The nature and probable origin of binaural beats. Psychological Review. 23 (5), 333-351.

61. Pigott, E., Alter, G., \& Marikis, D. (2015). Frequency-Based Light \& Sound Neurotherapy (LSN) Research: A Review of the Research. Retrieved on March 2015 from https://www.psio.com/pdf/frequency-based-light-and-sound-neurotherapy.pdf

62. Plasier, S.A., Bulut, M., Aarts, R.M. (2011). A study of monaural beat effect on brain activity using an electronic singing bowl. $10^{\text {th }}$ Belgian Day on Biomedical Engineering - joint meeting with IEEE EMBS Benelux Chapter.

63. Pratt, C.C. (1930). The spatial character of high and low tones. Journal of Experimental Psychology, 13, 278-85.

64. Pratt, H., Starr, A., Michalewski, H. J., Dimitrijevic, A., Bleich, N., \& Mittelman, N. (2010). A comparison of auditory evoked potentials to acoustic beats and to binaural beats. Hearing Research, 262, 34-44.

65. Rabipour, S., Raz, A. 2012. Training the brain: Fact and fad in cognitive and behavioral remediation. Brain and cognition. 79(2), 159-179.

66. Reedijk, S. A., Bolders, A., \& Hommel, B. (2013). The impact of binaural beats on creativity. Frontiers in human neuroscience, 7.

67. Ritskes, R., Ritskes-Hoitinga, A., \& Stødkilde-Jørgensen, A. (2004). MRI scanning during Zen meditation: The picture of enlightenment? The Relevance of the Wisdom Traditions in Contemporary Society: The Challenge to Psychology, 195.

68. Rosenberg, L. 1999. Breath by breath: The liberating practice of insight meditation. Boston: Shambhala.

69. Sanches, P., Höök, K., Vaara, E., Weymann, C., Bylund, M., Ferreira, P. Peria, N., Sjölinder, M. 2010. Mind the body!: designing a mobile stress management application encouraging personal reflection. In Proc. of the Designing Interactive Systems, 47-56. ACM.

70. Sas, C. (2007). Being Present - Being Mindful. Conceptual delimitations and comparative $\begin{array}{lllll}\text { analysis. } & \text { Proceeding } & \text { of } & \text { Peach }\end{array}$ http://www.peachbit.org/sites/peachbit.org/files/Corina_Sas_27122007.pdf

71.Sas, C. (2005). Sense of Presence. In Ghaoui, C. (Ed.) Encyclopedia of human computer interaction. IGI Global, 511-517

72. Sas, C., \& Dix, A. (2009). Designing for reflection on experience. In CHI'09 Extended Abstracts on Human Factors in Computing Systems (pp. 4741-4744). ACM. 
73. Sas, C., Fratczak, T., Rees, M., Gellersen, H., Kalnikaite, V., Coman, A., \& Höök, K. (2013). AffectCam: arousal-augmented Sensecam for richer recall of episodic memories. In CHI'13 Extended Abstracts on Human Factors in Computing Systems (pp. 1041-1046). ACM.

74. Schiphorst, T. (2011). Self-evidence: applying somatic connoisseurship to experience design. In Proc. Extended abstracts on Human factors in computing systems, 145-160). ACM.

75. Shaw, C., Gromala, D., Song, M. 2010. The meditation chamber: Towards self-modulation. Metaplasticity in Virtual Worlds: Aesthetics and Semantics Concepts, IGI Publishing.

76. Siever, D. 2007 Audio-visual entrainment: history, physiology, and clinical studies. Handbook of Neurofeedback: Dynamics and Clinical Applications, 155-183. Binghamton, NY: The Haworth Medical Press.

77. Speca, M., Carlson, L.E., Goodey, E., Angen, M. 2000. A randomized, wait-list controlled clinical trial: the effect of a mindfulness meditation-based stress reduction program on mood and symptoms of stress in cancer outpatients. Psychosomatic medicine, 62(5), 613-622.

78. Ståhl, A., Höök, K., Svensson, M., Taylor, A. S., Combetto, M. 2009. Experiencing the affective diary. Personal and Ubiquitous Computing, 13(5), 365-378.

79. Stinson, B., Arthur, D. 2013. A novel EEG for alpha brain state training, neurobiofeedback and behavior change. Complementary Therapies in Clinical Practice, 114-118.

80. Stytsenko, K., Jablonskis, E., \& Prahm, C. (2011, August). Evaluation of consumer EEG device Emotiv EPOC. In MEi: CogSci Conference 2011, Ljubljana.

81. Teasdale, J.D., Segal, Z.V., Williams, J.M.G., Ridgeway, V., Soulsby, J., Lau, M. 2000. Prevention of relapse/recurrence in major depression by mindfulness-based cognitive therapy. Journal of Consulting and Clinical Psychology, 6, 615-623).

82. Thieme, A., Wallace, J., Johnson, P., McCarthy, J., Lindley, S., Wright, P., Olivier, P. Meyer, T.D. 2013. Design to promote mindfulness practice and sense of self for vulnerable women in secure hospital services. In Proc. of the SIGCHI Conference on Human Factors in Computing Systems (CHI '13). ACM, 2647-2656.

83. Travis, F. 2001. Autonomic and EEG patterns distinguish transcending from other experiences during transcendental meditation practice. Int. J. Psychophysiol., 42, pp. 1-9

84. Valentine, E.R., Sweet, P.L. 1999. Meditation and attention: A comparison of the effects of concentrative and mindfulness meditation on sustained attention. Mental Health, Religion \& Culture, 2(1), 59-70.

85. Vernon, D. (2009). Human potential: Exploring techniques used to enhance human performance. Routledge.

86. Vernon, D., Peryer, G., Louch, J., \& Shaw, M. (2014). Tracking EEG changes in response to alpha and beta binaural beats. International Journal of Psychophysiology, 93(1), 134-139.

87. Vernon, D. (2005). Can neurofeedback training enhance performance? An evaluation of the evidence with implications for future research. Applied Psychophysiology and Biofeedback, 30(4), 347-364.

88. Vidyarthi, J., Riecke, B. E., Gromala, D. 2012. Sonic Cradle: designing for an immersive experience of meditation by connecting respiration to music. In Proc. of the Designing Interactive Systems Conference, 408-417. ACM.

89. Wang, D., Xu, M., Zhanq, Y., \& Xiao, J. (2013, April). Preliminary study on hapticstimulation based brainwave entrainment. In World Haptics Conference, 2013 (pp. 565-570). IEEE.

90. Weiser, M., Brown, J.S. 1996. Designing calm technology. PowerGrid Journal, 1(1), 75-85.

91. Yu, M. C., Wu, H., Lee, M. S., Hung, Y. P. 2012. Multimedia Assisted Breathwalk-aware System. IEEE Transaction on Biomedical Engineering, 59(12), 3276-3282

92.Zeidan, F., Gordon, N. S., Merchant, J., Goolkasian, P. (2010a). The effects of brief mindfulness meditation training on experimentally induced pain. Journal of Pain, 11(3), 199.

93. Zeidan, F., Johnson, S. K., Diamond, B. J., David, Z., Goolkasian, P. (2010b). Mindfulness meditation improves cognition: Evidence of brief mental training. Consciousness and cognition, 19(2), 597-605. 